\title{
Consensus-Based Recommendations for Titrating Cannabinoids and Tapering Opioids for Chronic Pain Control
}

\author{
Aaron Sihota ${ }^{1}$, Brennan Smith² ${ }^{2}$ Sana-Ara Ahmed ${ }^{3}$, Alan Bell ${ }^{4}$, Allison Blain ${ }^{5}$, Hance \\ Clarke $^{4}$, Ziva Cooper ${ }^{6}$, Claude Cyr $^{7}$, Paul Daeninck ${ }^{8}$, Amol Deshpande ${ }^{9}$, Karen Ethans ${ }^{8}$, \\ David Flusk $^{10}$, Bernard Le Foll ${ }^{11}$, M-J Milloy ${ }^{12}$, Dwight Moulin ${ }^{13}$, Vernon Naidoo ${ }^{14}$, May \\ Ong $^{15}$, Jordi Perez ${ }^{7}$, kevin ROD ${ }^{4}$, Robert Sealey ${ }^{16}$, Dustin Sulak ${ }^{17}$, Zachary Walsh ${ }^{15}$, and \\ Colleen O'Connell ${ }^{18}$ \\ ${ }^{1}$ The University of British Columbia Faculty of Pharmaceutical Sciences \\ ${ }^{2}$ CTC Communications Corp \\ ${ }^{3}$ Ahmed Institute for Pain and Cannabinoid Research \\ ${ }^{4}$ University of Toronto \\ ${ }^{5}$ McMaster University \\ ${ }^{6} \mathrm{UCLA}$ \\ ${ }^{7}$ McGill University \\ ${ }^{8}$ University of Manitoba \\ ${ }^{9}$ Toronto Rehabilitation Institute \\ ${ }^{10}$ Memorial University of Newfoundland \\ ${ }^{11}$ Centre for Addiction and Mental Health \\ ${ }^{12}$ British Columbia Centre on Substance Use \\ ${ }^{13}$ Western University \\ ${ }^{14}$ General Practitioner \\ ${ }^{15}$ The University of British Columbia \\ ${ }^{16}$ Cannabinoid Medicine Specialist \\ ${ }^{17}$ Integr8 Health \\ ${ }^{18}$ Stan Cassidy Centre for Rehabilitation
}

May 19, 2020

\begin{abstract}
Aims: Opioid misuse and overuse has contributed to a widespread overdose crisis and many patients and physicians are considering medical cannabis to support opioid tapering and chronic pain control. Using a five-step modified Delphi process, we aimed to develop consensus-based recommendations on: 1) when and how to safely initiate and titrate cannabinoids in the presence of opioids, 2) when and how to safely taper opioids in the presence of cannabinoids, and 3) how to monitor patients and evaluate outcomes when treating with opioids and cannabinoids. Results: In patients with chronic pain taking opioids not reaching treatment goals, there was consensus that cannabinoids may be considered for patients experiencing or displaying opioid-related complications, despite psychological or physical interventions. There was consensus observed to initiate with a CBD-predominant oral extract in the daytime and consider adding THC. When adding THC, start with $0.5-3$ mg, and increase by $1-2 \mathrm{mg}$ once or twice weekly up to $30-40 \mathrm{mg} /$ day. Initiate opioid tapering when the patient reports a minor/major improvement in function, seeks less as-needed medication to control pain, and/or the cannabis dose has been optimized. The opioid tapering schedule may be $5 \%-10 \%$ of the morphine equivalent dose (MED) every 1 to 4 weeks. Clinical success could be
\end{abstract}


defined by an improvement in function/quality of life, a [?] $30 \%$ reduction in pain intensity, a [?] $25 \%$ reduction in opioid dose, a reduction in opioid dose to $<90 \mathrm{mg} \mathrm{MED}$, and/or reduction in opioid-related adverse events. Conclusions: This five-stage modified Delphi process led to the development of consensus-based recommendations surrounding the safe introduction and titration of cannabinoids in concert with tapering opioids.

\section{Introduction}

Opioids are commonly prescribed for chronic pain management and although data support their role for managing acute and cancer-related pain, the evidence to support use in chronic pain is not robust. ${ }^{1-4}$ Prescription opioid misuse has contributed to a widespread opioid overdose crisis resulting in the deaths of hundreds of thousands of individuals worldwide. ${ }^{5,6}$

Medical cannabis containing different concentrations of cannabinoids - such as $\Delta^{9}$-tetrahydrocannabinol (THC) and cannabidiol (CBD) - is increasingly being used for the management of chronic pain. ${ }^{7,8}$ Cannabinoids have a lower risk for dependence compared with opioids and the predicted median lethal dose for THC is $>1000$ fold higher than the effective dose. ${ }^{9-11}$ Previous studies have found that cannabinoids can improve pain related-outcomes, quality of life and, importantly, have an opioid-sparing effect. ${ }^{7,12-19}$ In addition, it has been reported that patients commonly use medical cannabis as a substitute for opioid medication. ${ }^{20-23}$ However the effectiveness of cannabis substitution for opioids is not universally observed. ${ }^{24,25}$

Although these findings are noteworthy, the majority of clinical studies investigating cannabis and cannabinoids as substitutes or adjuncts to opioids are cross-sectional or small sample-size randomized controlled trials. This lack of high-quality evidence makes providing classical evidence-based recommendations inaccessible. Despite the paucity of clinical trial evidence, physicians and patients are using cannabis to support opioid tapering. In many countries, patients with chronic pain have access to cannabis, and patients have reported self-administering cannabis to reduce their opioid dose in the absence of clinical guidance. ${ }^{22,26,27}$

Although cannabis has a lower risk of dependence compared to opioids, it is not an inert therapy. ${ }^{28-31}$ At high doses, CBD-related side effects can include fatigue, diarrhea, and changes in appetite and weight. ${ }^{32}$ THC-related side effects can include sedation, syncope, tachycardia, risk of cannabis-use disorder, psychosis, and anxiety. ${ }^{8,33}$ With patients having access to prescribed cannabinoids and self-treating with cannabis to reduce their opioid dose, clinical guidance on safe cannabinoid initiation and titration is urgently required. Randomized placebo-controlled clinical trials examining how to co-manage cannabinoids and opioids are unlikely to be provided in the near future. Hence there is an immediate unmet need for guidance on this topic. ${ }^{34}$

To provide guidance to health care professionals on how to safely manage opioids and cannabinoids in patients with chronic pain, we employed a modified Delphi process to develop a consensus-based guidance algorithm. The modified Delphi process has been used extensively in health care settings to provide consensus-based recommendations surrounding important clinical questions. ${ }^{35}$ A previous Delphi study related to opioids and cannabis was undertaken between 2015 and 2016 and aimed to develop consensus guidelines for responding to patients on long term opioids using cannabis, however, the experts disagreed on many of the proposed topics. $^{36}$

The purpose of the present initiative was to develop consensus-based recommendations on 1) when and how to safely initiate and titrate cannabinoids in the presence of opioids, 2) when and how to safely taper opioids in the presence of cannabinoids, and 3) how to monitor patients and evaluate clinical outcomes when treating with opioids and cannabinoids.

\section{Methods}

\section{The Modified Delphi Process}

A modified Delphi process was used to develop this consensus guidance document and associated algorithm. ${ }^{37-39}$ The methods used for this report have been previously published in abstract form. ${ }^{40}$ The participants were recruited based on extensive clinical experience with prescribing and managing patients 
on medical cannabis and/or extensive research expertise with cannabis. The consensus process incorporated a five-step modified Delphi method similar to previous reports ${ }^{41-43}$ and took place between July 2019 and November 2019 (Figure 1 and Table 1). In step one a core scientific committee of cannabinoid subject matter experts from the United States and Canada $(\mathrm{n}=9)$ identified key areas of focus. From these areas of focus, an initial draft of consensus questions was developed, and these questions were incorporated into four domains:

1. When to consider introducing cannabinoids in patients with chronic pain taking opioids

2. How to introduce cannabinoids in patients with chronic pain taking opioids

3. When and how to taper opioids in patients with chronic pain taking cannabinoids

4. Evaluating clinical outcomes and guiding patient monitoring and safety

In step two the core scientific committee reviewed the initial draft of questions and provided comments. Following the inclusion of the suggested changes to the consensus questions, a teleconference was conducted to gain verbal approval from the scientific committee to send out the questions for review by the rest of the consensus summit participants $(\mathrm{n}=13)$.

In step three the consensus summit participants were provided a reference package and sent the consensus questions for their review and associated comments. Twelve of the 13 participants provided their comments and suggestions.

Following the inclusion of these updates into the consensus questions, step four was initiated and all summit invitees, which included the core scientific committee and the participants, reviewed the consensus questions and prevoted using an online software. Sixteen of the 22 summit invitees provided a prevote. These prevote results were then used at the live event to focus the discussion on topics where a lack of consensus was apparent. In step five a formal voting session took place at an in-person meeting in Toronto, Canada: The Opioids and Cannabinoids Consensus Summit. The voting was public but anonymous using live polling software (Slido, www.slido.com). Nineteen participants took part in the live voting session, however the opportunity to abstain from answering questions was available.

For consensus to be declared, a predetermined threshold of [?] $75 \%$ of the voters had to agree on a specific answer, or, [?] $75 \%$ of the voters had to strongly agree or agree (or strongly disagree or disagree) on an answer. This consensus threshold is similar to previous studies using a modified Delphi method. ${ }^{41,44}$ At the in-person event, revisions to the questions and associated answers, and revotes, were permitted. The voters were instructed that the patient they were considering was a patient with chronic pain taking opioids who was not currently using cannabis, recreationally or medically, to treat their chronic pain. The voters were instructed that the termcannabinoids refers to the most studied of the cannabinoids, ie, THC and CBD. The voters were instructed to assume there were no patient access or financial limitations to consider when choosing a given answer.

Results

The results described below highlight what we believe are the key consensus findings. The full complement of questions and answers are available in the Supplementary Tables. A treatment guidance algorithm for health care professionals was developed based on the answers where consensus was reached (Figure 2).

\section{Domain 1: When to consider introducing cannabinoids in patients with chronic pain taking opioids}

The first domain asked questions about patient factors that may influence the suitability of a patient for treatment with cannabinoids. The key consensus findings are:

1) If a patient has a history of psychosis, is pregnant or breastfeeding, or has had an adverse reaction to cannabinoids, cannabinoids should be avoided. Although, when considering a patient with a history of psychosis, THC appears to be the more significant causal agent and CBD may in fact reduce psychosis. ${ }^{45,46}$ Physicians may consider medical cannabis in a patient: taking opioids at any morphine equivalent dose 
(MED), not reaching chronic pain goals, experiencing opioid-related adverse effects, and/or, displaying risk factors for opioid-related harm. It is important to note that this consensus initiative does not aim to suggest that all patients taking opioids should reduce their opioids. In a recent commentary, the Centre for Disease Control guidelines for opioid tapering were clarified to highlight that clinicians should avoid increasing the opioid dosage to [?] $90 \mathrm{MED}$, but not necessarily discontinue opioids in patients on a high dose. ${ }^{47}$ Dialogue and shared-decision making with the patient and carefully evaluating the benefits and risks associated with tapering and discontinuation of opioids is strongly encouraged. In addition, before the introduction of any additional pharmaceutical intervention, evidence-based psychological and physical therapy interventions to reduce opioid use should be attempted and maximized. ${ }^{48,49}$

2) There was consensus that no age restrictions were recommended for CBD or THC use. With respect to $\mathrm{CBD}$, the group quickly reached consensus as high doses of CBD have been shown to be safe in children, albeit in a patient population dissimilar to typical patients with chronic pain. ${ }^{50,51}$ Conversely, there was debate surrounding the minimum age recommendation for THC. When using THC, careful consideration in the younger population should be made as the nervous system is not fully developed until 25 years of age. ${ }^{52}$ However, the consensus summit participants debated that if a young patient is already on opioids it did not seem rational to withhold cannabinoid therapy until they turn a specific age. Similarly, although there was no maximum age agreed to, careful consideration must be given when considering cannabinoids to the elderly, while also recognizing that the elderly are particularly susceptible to the adverse effects of opioids.

\section{Domain 2: How to introduce cannabinoids in patients with chronic pain taking opioids}

The second domain asked questions regarding how to administer, initiate, titrate, and dose cannabinoids in the presence of opioids. The key consensus findings are:

1) There was consensus that the preferred format of administering cannabinoids was the oral route, using oil extracts or capsules. Sublingual tinctures may also be considered. There was consensus strongly recommending against smoking cannabis. Caution should be used in jurisdictions where regulated and standardized medical cannabis products are unavailable.

2) In the daytime, it was agreed that a patient should initiate with CBD oral dosing at a range of 5-20 mg. For THC, there was consensus that the initial THC dose range should be $0.5-3 \mathrm{mg}$ and then titrated up by 1-2 mg every one or two weeks to reach chronic pain goals.

This initiation and titration protocol deserves further discussion. Cannabidiol was recommended as the initiating cannabinoid for daytime dosing partly because there is limited sedation or intoxication associated with CBD. ${ }^{53}$ In addition, CBD unaccompanied by THC has been observed to support opioid tapering and reduce cue-induced cravings for opioids. ${ }^{54,55}$ Published CBD doses range from tens of $\mathrm{mg}$ to thousands of mg yet from a practical standpoint, the cost of CBD may become a limitation at high doses. As such there was no consensus on how high up the CBD dose should be titrated. It is important to note that the available evidence for CBD alone to improve chronic pain control is weak. ${ }^{56-59}$ Preliminary studies examining topical application of $\mathrm{CBD}$ have observed that this route may be efficacious for treating pain, although further research is required. ${ }^{60,61}$

In contrast to $\mathrm{CBD}$, the available evidence for THC to improve chronic pain control is relatively strong. ${ }^{7,62}$ During the consensus summit, it was noted that some patients can reach pain control goals with CBD alone, but it was quickly pointed out that many CBD preparations, both medical and recreational, contain a small percentage of THC and thus it could be the low concentration of THC providing the pain relief associated with CBD, especially at high CBD doses. Health care professionals may consider adding THC soon after CBD initiation if the patient is not reaching their chronic pain control goals.

The initiating dose range of $0.5-3 \mathrm{mg}$ THC was chosen based on clinical observations that patients typically begin to experience psychoactive effects at $2-2.5 \mathrm{mg}$ of THC, which is similar to the reported effective dose of dronabinol, a synthetic isomer of THC, ${ }^{63}$ and many patients would prefer to avoid the psychoactive effect. 
In addition, there is individual variability regarding the response to THC, and taking a "start low and go slow" approach is recommended. There are also reported sex differences in the response to THC as a recent placebo-controlled study observed that women experience similar psychoactive effects at a lower THC dose in comparison to men. ${ }^{64}$ Our consensus findings on how to initiate and titrate cannabinoids in the presence of opioids are similar to a previously published editorial on cannabinoid dosing for chronic pain management. ${ }^{65}$

A caveat to the "start low and go slow" approach may be considered with a patient at high risk for opioidrelated harm. The need to rapidly increase cannabinoids may be apparent as the opioid taper may need to be more aggressive. Therefore, under certain circumstances, it may be appropriate to start low but go fast with cannabinoid introduction and titration. Patient-specific factors such as response and access to close monitoring may allow for a more aggressive cannabinoid titration and be a valuable strategy in a patient where the opioid risks are high.

3) For night-time use, there was no consensus on the CBD or THC dose, or the THC:CBD distribution ratio, although there was discussion around the potential importance of THC for sleep quality, which may support chronic pain relief. ${ }^{66}$

4) For breakthrough pain, vapourization of dried cannabis flower was recommended. It is important to note that vapourizing dried flower differs from vaping through an electronic cigarette device. Vapourization of dried flower is ideally accomplished with an approved medical devices, although many different types of vapourizers of differing quality could be used ${ }^{67}$ In contrast, vaping cannabis using electronic cigarette devices may expose the patient to unsafe additives and increase the risk of a novel lung disease, EVALI (e-cigarette or vaping product use associated lung injury). ${ }^{68,69}$ Until such a time that electronic cigarette devices are proven safe, inhalation of medical cannabis for breakthrough pain should be undertaken exclusively with medically-approved vapourizers using dried cannabis flowers.

\section{Domain 3: When and how to taper opioids in patients with chronic pain taking cannabinoids}

The third domain discussed questions around when and how to taper opioids in patients being administered opioids and cannabinoids. The key consensus findings are:

1) Depending on the patient, begin the opioid taper when any of the following criteria are met: the patient has an improvement in pain/function, the cannabinoid dose has been optimized, or the patient begins to seek less "as needed" medication. Importantly, it was recommended to not begin tapering opioids at cannabinoid initiation or a specific cannabinoid dose.

2) When initiating the opioid taper, a gradual opioid dose reduction of $5-10 \%$ of the MED every one to four weeks was agreed upon. The timeline for the frequency of dose reduction is broad to allow for patient tailoring, and is similar to previously published opioid tapering recommendations. ${ }^{1,3}$ More recently, the U.S. Department of Health \& Human Services have published opioid-tapering guidelines. ${ }^{70,71}$ These guidelines do not provide specific MED targets and encourage a highly individualized opioid tapering approach and collaborative shared decision-making with the patient. Indeed, there may be patients who could benefit from a 20\%-50\% rapid taper after titrating to an effective cannabinoid dose, depending on their objectives and needs.

\section{Domain 4: Evaluating clinical outcomes and guiding patient monitoring and safety}

The fourth domain examined questions around monitoring, safety, and efficacy measures in patients with chronic pain taking opioids and cannabinoids. The key consensus findings for this domain are:

1) During the early phase of opioids and cannabinoids co-administration, it was recommended to follow-up with patients once or twice monthly until the patient is stable. Once the health care professional and the patient are comfortable, it was agreed that follow-up could occur every three months. It was also agreed that the monitoring of these patients could be led by health care professionals other than the treating physician, and that these follow-ups could be done via phone call or home visit depending on what works best for the health care professional and patient. At this point, even though a patient may be stable with respect to 
their chronic pain control, variations in dosing and administration of medical cannabis may be required for optimal pain control.

2) When considering the safety of a patient taking both opioids and cannabinoids, health care professionals should screen for opioid withdrawal, illicit opioid use, cannabinoid-related adverse effects, use of other illicit substances, and symptoms of psychosis. Cannabinoid titration should be halted: when the patient's goals are met; when cannabinoid treatment reaches an efficacy plateau (ie, no change in pain relief after a dose increase); or, if the patient experiences a cannabinoid-related adverse event. If the patient experiences a cannabinoid-related adverse event, it is recommended to reduce the dose of the associated cannabinoid. If a patient experiences opioid withdrawal symptoms, it was agreed that the health care professional should consider slowing or pausing the patient's opioid taper.

3) When considering treatment success, no consensus was found on the need to use a validated questionnaire to establish efficacy. However, there was consensus on initiating and documenting discussions with the patient around the degree of pain relief, sleep quality, and everyday functionality throughout treatment. In concert with this, it was agreed that clinical success when titrating cannabinoids and tapering opioids is most clearly defined by an improvement in patient function.

\section{Discussion}

Globally, cannabis is being used to support a reduction or cessation of opioid use for pain. This utilization of cannabis is occurring despite a lack of placebo-controlled, randomized clinical trials and highlights a practical unmet need for expert guidance on the safe co-management of cannabinoids and opioids. The aim of the present project was to develop consensus-based recommendations on how to safely and effectively manage cannabinoid initiation and titration with opioid tapering. The primary consensus findings are provided as an algorithm (Figure 2) to be applied in clinical practice by the health care team.

An important stipulation when considering these consensus-based recommendations is that a patient's personal considerations should always be taken into account, and that the treating physician's clinical rationale and individual assessment of the patient is paramount. Many of the consensus recommendations are presented as ranges to allow the health care professional to tailor the cannabinoid and opioid management strategy on an individual basis. Additionally, it is important to maximize psychological and physical therapy treatment interventions before initiation of cannabinoids or any additional pharmaceutical therapy.

A limitation of the consensus-based recommendations provided herein is that they are based primarily on expert opinion developed through a modified Delphi process, and not placebo-controlled, randomized clinical trials. However, as there is a lack of high-quality literature investigating opioids and medical cannabis, the authors of this document leveraged their real-world experience across tens of thousands of patient interactions to support the development of this guidance algorithm with a focus on safety. As new evidence becomes available related to the use of cannabinoids and opioids in patients with chronic pain, the recommendations made within this document will be updated and refined.

We also note that the education of health care professionals surrounding the safe and effective use of medical cannabis is lacking. ${ }^{72,73}$ We hope that the introduction of this algorithm will initiate more extensive conversations about key educational needs surrounding the safe and effective use of medical cannabis, which may include focused dialogue on the divergent, but perhaps complementary, physiological effects of CBD and THC that lead to the support of opioid tapering and improved pain control. We would recommend learning modules on chronic pain treatment with medical cannabis be completed before prescribing permission be granted.

The unmet need for expert guidance on co-managing cannabinoids and opioids prompted the development of this consensus-based document. Additionally, in the midst of an opioid crisis, the validation of strategies to reduce exposure to opioids is of interest for public health policy. We employed a modified Delphi process to add robustness to our scientific process as these expert consensus-based recommendations are derived primarily from real-world clinical experience in lieu of placebo-controlled, randomized clinical trials. This 
modified Delphi process led to the development of a series of consensus-based recommendations surrounding the introduction and titration of cannabinoids in concert with the tapering of opioids. These recommendations should be evaluated prospectively to examine if target reductions in opioids may be met. Future rigorous experimental studies in this area will be integral in helping shape formal guidelines.

\section{References}

1. Dowell D, Haegerich TM, Chou R. CDC guideline for prescribing opioids for chronic pain - United States, 2016. MMWR Recomm Reports . 2016;65(1):1-49. doi:10.15585/mmwr.rr6501e1

2. Chou R, Turner JA, Devine EB, et al. The effectiveness and risks of long-term opioid therapy for chronic pain: A systematic review for a national institutes of health pathways to prevention workshop. Ann Intern Med . 2015;162(4):276-286. doi:10.7326/M14-2559

3. Busse JW, Craigie S, Juurlink DN, et al. Guideline for opioid therapy and chronic noncancer pain. CMAJ . 2017;189(18):E659-E666. doi:10.1503/cmaj.170363

4. Inturrisi CE. Clinical Pharmacology of Opioids for Pain .; 2002.

5. WHO | Information sheet on opioid overdose. WHO . 2018.

6. Han B, Compton WM, Blanco C, Crane E, Lee J, Jones CM. Prescription opioid use, misuse, and use disorders in U.S. Adults: 2015 national survey on drug use and health. Ann Intern Med . 2017;167(5):293301. doi:10.7326/M17-0865

7. The Health Effects of Cannabis and Cannabinoids: The Current State of Evidence and Recommendations for Research : Health and Medicine Division. http://www.nationalacademies.org/hmd/Reports/2017/health-effects-of-cannabis-and-cannabinoids.aspx. Accessed December 1, 2019.

8. (No Title). https://www.canada.ca/content/dam/hc-sc/documents/services/drugsmedication/cannabis/information-medical-practitioners/information-health-care-professionals-cannabiscannabinoids-eng.pdf. Accessed December 1, 2019.

9. Thompson GR, Rosenkrantz H, Schaeppi UH, Braude MC. Comparison of acute oral toxicity of cannabinoids in rats, dogs and monkeys. Toxicol Appl Pharmacol . 1973;25(3):363-372. doi:10.1016/0041008X(73)90310-4

10. Ware MA, St Arnaud-Trempe E. The abuse potential of the synthetic cannabinoid nabilone. Addiction . 2010;105(3):494-503. doi:10.1111/j.1360-0443.2009.02776.x

11. Katsidoni V, Anagnostou I, Panagis G. Cannabidiol inhibits the reward-facilitating effect of morphine: Involvement of 5-HT1A receptors in the dorsal raphe nucleus. Addict Biol . 2013;18(2):286-296. doi:10.1111/j.1369-1600.2012.00483.x

12. Cooper ZD, Bedi G, Ramesh D, Balter R, Comer SD, Haney M. Impact of co-administration of oxycodone and smoked cannabis on analgesia and abuse liability. Neuropsychopharmacology . 2018. doi:10.1038/s41386018-0011-2

13. Nielsen S, Sabioni P, Trigo JM, et al. Opioid-sparing effect of cannabinoids: A systematic review and meta-analysis.Neuropsychopharmacology . 2017. doi:10.1038/npp.2017.51

14. Lake S, Walsh Z, Kerr T, et al. Frequency of cannabis and illicit opioid use among people who use drugs and report chronic pain: A longitudinal analysis. Alegria M, ed. PLOS Med . 2019;16(11):e1002967. doi:10.1371/journal.pmed.1002967

15. Abrams DI, Couey P, Shade SB, Kelly ME, Benowitz NL. Cannabinoid-opioid interaction in chronic pain. Clin Pharmacol Ther . 2011;90(6):844-851. doi:10.1038/clpt.2011.188 
16. Ware MA, Wang T, Shapiro S, Collet JP. Cannabis for the Management of Pain: Assessment of Safety Study (COMPASS). J Pain . 2015;16(12):1233-1242. doi:10.1016/j.jpain.2015.07.014

17. Haroutounian S, Ratz Y, Ginosar Y, et al. The Effect of Medicinal Cannabis on Pain and Qualityof-Life Outcomes in Chronic Pain: A Prospective Open-label Study. Clin J Pain . 2016;32(12):1036-1043. doi:10.1097/AJP.0000000000000364

18. Sagy I, Bar-Lev Schleider L, Abu-Shakra M, Novack V. Safety and Efficacy of Medical Cannabis in Fibromyalgia. J Clin Med . 2019;8(6):807. doi:10.3390/jcm8060807

19. Rod K. A Pilot Study of a Medical Cannabis - Opioid Reduction Program. Am J Psychiatry Neurosci . 2019;7(3):74. doi:10.11648/j.ajpn.20190703.14

20. Abuhasira R, Schleider LBL, Mechoulam R, Novack V. Epidemiological characteristics, safety and efficacy of medical cannabis in the elderly.Eur J Intern Med . 2018;49:44-50. doi:10.1016/j.ejim.2018.01.019

21. Lucas P, Walsh Z. Medical cannabis access, use, and substitution for prescription opioids and other substances: A survey of authorized medical cannabis patients. Int J Drug Policy . 2017;42:30-35. doi:10.1016/j.drugpo.2017.01.011

22. Lucas P, Baron EP, Jikomes N. Medical cannabis patterns of use and substitution for opioids \&amp; other pharmaceutical drugs, alcohol, tobacco, and illicit substances; results from a cross-sectional survey of authorized patients. Harm Reduct J . 2019;16(1):9. doi:10.1186/s12954-019-0278-6

23. Lucas P, Walsh Z, Crosby K, et al. Substituting cannabis for prescription drugs, alcohol and other substances among medical cannabis patients: The impact of contextual factors. Drug Alcohol Rev . 2016;35(3):326-333. doi:10.1111/dar.12323

24. Wildes M, Bigand TL, Layton ME, Wilson M. Cannabis Use and Cognition in Adults Prescribed Opioids for Persistent Pain. Pain Manag Nurs . 2019. doi:10.1016/j.pmn.2019.06.014

25. Campbell G, Hall WD, Peacock A, et al. Effect of cannabis use in people with chronic non-cancer pain prescribed opioids: findings from a 4-year prospective cohort study. Lancet Public Heal . 2018;3(7):e341e350. doi:10.1016/S2468-2667(18)30110-5

26. Reiman A, Welty M, Solomon P. Cannabis as a Substitute for Opioid-Based Pain Medication: Patient Self-Report. doi:10.1089/can.2017.0012

27. Boehnke KF, Litinas E, Clauw DJ. Medical Cannabis Use Is Associated with Decreased Opiate Medication Use in a Retrospective Cross-Sectional Survey of Patients with Chronic Pain. J Pain . 2016;17(6):739744. doi:10.1016/j.jpain.2016.03.002

28. Hamilton I, Monaghan M. Cannabis and Psychosis: Are We any Closer to Understanding the Relationship? Curr Psychiatry Rep . 2019;21(7). doi:10.1007/s11920-019-1044-x

29. Lev-Ran S, Roerecke M, Le Foll B, George TP, McKenzie K, Rehm J. The association between cannabis use and depression: A systematic review and meta-analysis of longitudinal studies. Psychol Med . 2014;44(4):797-810. doi:10.1017/S0033291713001438

30. Fischer B, Russell C, Sabioni P, et al. Lower-Risk Cannabis Use Guidelines: A Comprehensive Update of Evidence and Recommendations.Am J Public Health . 2017;107(8):e1-e12. doi:10.2105/AJPH.2017.303818

31. Volkow ND, Baler RD, Compton WM, Weiss SRB. Adverse health effects of marijuana use. $N$ Engl $J$ Med . 2014;370(23):2219-2227. doi:10.1056/NEJMra1402309

32. Iffland K, Grotenhermen F. An Update on Safety and Side Effects of Cannabidiol: A Review of Clinical Data and Relevant Animal Studies.Cannabis Cannabinoid Res . 2017;2(1):139-154. doi:10.1089/can.2016.0034 
33. Whiting PF, Wolff RF, Deshpande S, et al. Cannabinoids for medical use: A systematic review and meta-analysis. JAMA - J Am Med Assoc . 2015;313(24):2456-2473. doi:10.1001/jama.2015.6358

34. Russo EB. Cannabis and Pain. Pain Med . September 2019. doi:10.1093/pm/pnz227

35. Hasson F, Keeney S, McKenna H. Research guidelines for the Delphi survey technique. J Adv Nurs . 2000;32(4):1008-1015. doi:10.1046/j.1365-2648.2000.t01-1-01567.x

36. Starrels JL, Young SR, Azari SS, et al. Disagreement and Uncertainty Among Experts About how to Respond to Marijuana Use in Patients on Long-term Opioids for Chronic Pain: Results of a Delphi Study.Pain Med . August 2019. doi:10.1093/pm/pnz153

37. Dalkey N. An experimental study of group opinion: The Delphi method.Futures . 1969. doi:10.1016/S0016-3287(69)80025-X

38. Dalkey N, Helmer O. An Experimental Application of the DELPHI Method to the Use of Experts. Manage Sci . 1963. doi:10.1287/mnsc.9.3.458

39. Saad F, Canil C, Finelli A, et al. A Canadian consensus forum on the management of patients with advanced prostate cancer. Can Urol Assoc J . 2019;14(4). doi:10.5489/cuaj.6082

40. PAINWeek 2019 Accepted Abstracts by PAINWeek - issuu. https://issuu.com/painweek/docs/accepted_full_abstracts_by_poster_number_8.26.19. Accessed December 16, 2019.

41. Gillessen S, Attard G, Beer TM, et al. Management of Patients with Advanced Prostate Cancer: The Report of the Advanced Prostate Cancer Consensus Conference APCCC 2017 [Figure presented]. In:European Urology . ; 2018. doi:10.1016/j.eururo.2017.06.002

42. Eubank BH, Mohtadi NG, Lafave MR, et al. Using the modified Delphi method to establish clinical consensus for the diagnosis and treatment of patients with rotator cuff pathology. BMC Med Res Methodol . 2016;16(1). doi:10.1186/s12874-016-0165-8

43. Stewart D, Gibson-Smith K, MacLure K, et al. A modified Delphi study to determine the level of consensus across the European Union on the structures, processes and desired outcomes of the management of polypharmacy in older people. Leong C, ed. PLoS One . 2017;12(11):e0188348. doi:10.1371/journal.pone.0188348

44. Diamond IR, Grant RC, Feldman BM, et al. Defining consensus: A systematic review recommends methodologic criteria for reporting of Delphi studies. J Clin Epidemiol . 2014;67(4):401-409. doi:10.1016/j.jclinepi.2013.12.002

45. McGuire P, Robson P, Cubala WJ, et al. Cannabidiol (CBD) as an adjunctive therapy in schizophrenia: A multicenter randomized controlled trial. Am J Psychiatry . 2018;175(3):225-231. doi:10.1176/appi.ajp.2017.17030325

46. Boggs DL, Surti T, Gupta A, et al. The effects of cannabidiol (CBD) on cognition and symptoms in outpatients with chronic schizophrenia a randomized placebo controlled trial. Psychopharmacology (Berl) . 2018;235(7):1923-1932. doi:10.1007/s00213-018-4885-9

47. Dowell D, Haegerich T, Chou R. No Shortcuts to Safer Opioid Prescribing. $N$ Engl J Med . 2019;380(24):2285-2287. doi:10.1056/NEJMp1904190

48. Tick H, Nielsen A, Pelletier KR, et al. Evidence-Based Nonpharmacologic Strategies for Comprehensive Pain Care: The Consortium Pain Task Force White Paper. Explore . 2018;14(3):177-211. doi:10.1016/j.explore.2018.02.001

49. PAIN MANAGEMENT BEST PRACTICES INTER-AGENCY TASK FORCE REPORT .; 2019. 
50. Devinsky O, Cross JH, Laux L, et al. Trial of Cannabidiol for Drug-Resistant Seizures in the Dravet Syndrome. N Engl J Med . 2017;376(21):2011-2020. doi:10.1056/NEJMoa1611618

51. Devinsky O, Patel AD, Cross JH, et al. Effect of Cannabidiol on Drop Seizures in the Lennox-Gastaut Syndrome. N Engl J Med . 2018;378(20):1888-1897. doi:10.1056/NEJMoa1714631

52. Arain M, Haque M, Johal L, et al. Maturation of the adolescent brain. Neuropsychiatr Dis Treat . 2013;9:449-461. doi:10.2147/NDT.S39776

53. Dalton WS, Martz R, Lemberger L, Rodda BE, Forney RB. Influence of cannabidiol on delta-9tetrahydrocannabinol effects. Clin Pharmacol Ther . 1976;19(3):300-309. doi:10.1002/cpt1976193300

54. Capano A, Weaver R, Burkman E. Evaluation of the effects of CBD hemp extract on opioid use and quality of life indicators in chronic pain patients: a prospective cohort study. Postgrad Med . November 2019:1-6. doi:10.1080/00325481.2019.1685298

55. Hurd YL, Spriggs S, Alishayev J, et al. Cannabidiol for the reduction of cue-induced craving and anxiety in drug-abstinent individuals with heroin use disorder: A double-blind randomized placebo-controlled trial. Am J Psychiatry . 2019;176(11):911-922. doi:10.1176/appi.ajp.2019.18101191

56. Li X, Vigil JM, Stith SS, Brockelman F, Keeling K, Hall B. The effectiveness of self-directed medical cannabis treatment for pain. Complement Ther Med . 2019;46:123-130. doi:10.1016/j.ctim.2019.07.022

57. Stith SS, Vigil JM, Brockelman F, Keeling K, Hall B. The Association between Cannabis Product Characteristics and Symptom Relief. Sci Rep . 2019;9(1). doi:10.1038/s41598-019-39462-1

58. Wade DT, Makela P, Robson P, House H, Bateman C. Do cannabis-based medicinal extracts have general or specific effects on symptoms in multiple sclerosis? A double-blind, randomized, placebo-controlled study on 160 patients. Mult Scler . 2004;10(4):434-441. doi:10.1191/1352458504ms1082oa

59. Wade DT, Robson P, House H, Makela P, Aram J. A preliminary controlled study to determine whether whole-plant cannabis extracts can improve intractable neurogenic symptoms. Clin Rehabil . 2003;17(1):2129. doi:10.1191/0269215503cr581oa

60. Hunter D, Oldfield G, Tich N, Messenheimer J, Sebree T. Synthetic transdermal cannabidiol for the treatment of knee pain due to osteoarthritis. Osteoarthr Cartil . 2018;26:S26. doi:10.1016/j.joca.2018.02.067

61. Xu DH, Cullen BD, Tang M, Fang Y. The Effectiveness of Topical Cannabidiol Oil in Symptomatic Relief of Peripheral Neuropathy of the Lower Extremities. Curr Pharm Biotechnol . 2019;20. doi:10.2174/1389201020666191202111534

62. Andreae MH, Carter GM, Shaparin N, et al. Inhaled Cannabis for Chronic Neuropathic Pain: A Meta-analysis of Individual Patient Data.J Pain . 2015;16(12):1221-1232. doi:10.1016/j.jpain.2015.07.009

63. Beal JE, Olson R, Laubenstein L, et al. Dronabinol as a treatment for anorexia associated with weight loss in patients with AIDS. J Pain Symptom Manage . 1995;10(2):89-97. doi:10.1016/0885-3924(94)00117-4

64. Matheson J, Sproule B, Di Ciano P, et al. Sex differences in the acute effects of smoked cannabis: evidence from a human laboratory study of young adults. Psychopharmacology (Berl) . 2019. doi:10.1007/s00213019-05369-y

65. Boehnke KF, Clauw DJ. Brief commentary: Cannabinoid dosing for chronic pain management. Ann Intern Med . 2019;170(2):118. doi:10.7326/M18-2972

66. Kuhathasan N, Dufort A, MacKillop J, Gottschalk R, Minuzzi L, Frey BN. The use of cannabinoids for sleep: A critical review on clinical trials. Exp Clin Psychopharmacol . 2019;27(4):383-401. doi:10.1037/pha0000285

67. Manwell LA, Charchoglyan A, Brewer D, Matthews BA, Heipel H, Mallet PE. A vapourized $69-$ tetrahydrocannabinol ( $\delta 9-\mathrm{THC})$ delivery system part I: Development and validation of a pulmonary cannabi- 
noid route of exposure for experimental pharmacology studies in rodents. J Pharmacol Toxicol Methods . 2014;70(1):120-127. doi:10.1016/j.vascn.2014.06.006

68. Layden JE, Ghinai I, Pray I, et al. Pulmonary Illness Related to E-Cigarette Use in Illinois and Wisconsin — Preliminary Report. N Engl J Med . September 2019. doi:10.1056/nejmoa1911614

69. Kalininskiy A, Bach CT, Nacca NE, et al. E-cigarette, or vaping, product use associated lung injury (EVALI): case series and diagnostic approach. Lancet Respir Med . 2019;7(12):1017-1026. doi:10.1016/S2213-2600(19)30415-1

70. Rubin R. HHS Guide for Tapering or Stopping Long-term Opioid Use.JAMA . 2019;322(20):1947. doi:10.1001/jama.2019.18979

71. CDC. HHS Guide for Clinicians on the Appropriate Dosage Reduction or Discontinuation of Long-Term Opioid Analgesics .

72. Morris NP. Educating Physicians about Marijuana. JAMA Intern Med . 2019;179(8):1017-1018. doi:10.1001/jamainternmed.2019.1529

73. Evanoff AB, Quan T, Dufault C, Awad M, Bierut LJ. Physicians-in-training are not prepared to prescribe medical marijuana.Drug Alcohol Depend . 2017;180:151-155. doi:10.1016/j.drugalcdep.2017.08.010

Tables

Table 1: Participation During the Modified Delphi Process

Modified Delphi Process Development Consensus Question Development

AS, BKS, CO AS, BKS, MJM, ZDC, HC, BLF, JP, KR, DS, RS, MO, SAA, CC, PD, KE, AD, I

Figure Legends:

Figure 1: Flow of Modified Delphi Process

Figure 2: Consensus-Based Algorithm for Titrating Cannabinoids and Tapering Opioids in Patients with Chronic Pain

\section{Hosted file}

Figure 1 2020-05-18.pptx available at https://authorea.com/users/323981/articles/452386consensus-based-recommendations-for-titrating-cannabinoids-and-tapering-opioids-forchronic-pain-control

\section{Hosted file}

Figure 2 2020-05-18.pptx available at https://authorea.com/users/323981/articles/452386consensus-based-recommendations-for-titrating-cannabinoids-and-tapering-opioids-forchronic-pain-control 